\title{
Cardiac rupture in takotsubo cardiomyopathy treated surgically
}

\author{
Małgorzata Zalewska-Adamiec ${ }^{1}$, Hanna Bachórzewska-Gajewska1,2, Marcin Kożuch ${ }^{1}$, Marek Frank³ \\ Tomasz Hirnle ${ }^{3}$, Sławomir Dobrzycki ${ }^{1}$
}

${ }^{1}$ Department of Invasive Cardiology, Medical University of Bialystok, Bialystok, Poland

${ }^{2}$ Department of Clinical Medicine, Medical University of Bialystok, Bialystok, Poland

${ }^{3}$ Department of Cardiac Surgery, Medical University of Bialystok, Bialystok, Poland

Adv Interv Cardiol 2016; 12, 3 (45): 278-279

DOI: 10.5114 /aic.2016.61655

A 74-year-old woman, without a history of cardiological problems or risk factors of cardiovascular diseases, was admitted to the emergency room of our hospital after $2 \mathrm{~h}$ of chest pain. During the transport, the patient received $5000 \mathrm{U}$ of unfractionated heparin, aspirin (300 mg) and clopidogrel (600 mg). The patient had suffered from an anxiety syndrome for several years.

At admission, the patient was in a serious condition generally, was vomiting, and had severe chest pain with signs of cardiogenic shock (skin pale and wet, blood presure (BP) and heart rate (HR) undetectable). Electrocardiogram demonstrated a sinus rhythm of 58 per minute with QS complex and ST segment elevation in precordial leads (V2-V6). Laboratory results revealed increased troponin I concentration $(2.041 \mathrm{ng} / \mathrm{ml})$. Following hemodynamic stabilization, the patient was transported to the catheterization laboratory. Coronarography did not reveal any significant stenosis. Left ventricle angiography (LVA) showed normal volume with contractile disturbances of apex and hyperkinesis of the basement segments, with ejection fraction (EF) of $56 \%$. Contrast outflow to the epicardium was observed within the area of the apex, through the perforated wall of the left ventricle (Figures $1 \mathrm{~A}-\mathrm{C})$. Echo confirmed the presence of fluid in the pericardium and cardiac tamponade. The patient was supported with intra-aortic balloon contra-pulsation and transported to the cardiac surgery for urgent intervention. During transport, the patient lost consciousness. After urgent cardiac tamponade decompression, the pulse and arterial pressure increased. Active bleeding through the ruptured left ventricle was observed in the area of the apex during the operation. Left ventricular plication with sutures on a double layered Teflon pad was performed.
The lines of the sutures were conducted through healthy tissues. Examination of the supported ruptured region showed left ventricle tightness and complete hemostasis. No significant complications were observed during the perioperative period. On the first day after the operation the patient was extubated, and on the fifth day the intra-aortic balloon contra-pulsation was removed. The patient was transported to the regional hospital on day 11 to continue the therapeutic and rehabilitation procedures. A discharge echocardiogram revealed akinesis of the apex and hypokinesis of the septum, with an EF of $50 \%$. The patient was under cardiosurgical follow-up for the next 3 months. She was in good general condition without any chest pain, and the wounds from the sternotomy were healing properly. In a 2.5-year long study the patient was in good condition and the echocardiogram conformed normokinesis of the left ventricle apex. She has been treated with bisoprolol $5 \mathrm{mg}$ and aspirin $75 \mathrm{mg}$ since hospitalization.

Most cases of takotsubo cardiomyopathy (TTC) have a good prognosis. However, occasionally the clinical outcome is complicated by cardiogenic shock, serious ventricular rhythm abnormalities or even cardiac rupture [13]. The mechanism of cardiac rupture in TTC is not as well understood as the causes of mechanical complications in other cases without significant coronary artery stenosis, i.e. dissolving thrombus, embolization, vessel spasm and myocardial bridging [4]. According to Kumar et al. [1] the risk factors for cardiac rupture in takotsubo patients are female gender, older age, persistent ST elevation, ST elevations in inferior leads (especially in II), higher systolic blood pressure and diastolic blood pressure, low EF and LV peak systolic pressure.

\section{Corresponding author:}

Małgorzata Zalewska-Adamiec MD, PhD, Department of Invasive Cardiology, Medical University of Bialystok, 24 A Skłodowskiej St, 15-276 Bialystok, Poland, phone: +48 603784 468, e-mail: mzalewska5@wp.pl

Received: 1.10.2015, accepted: 5.12.2015. 

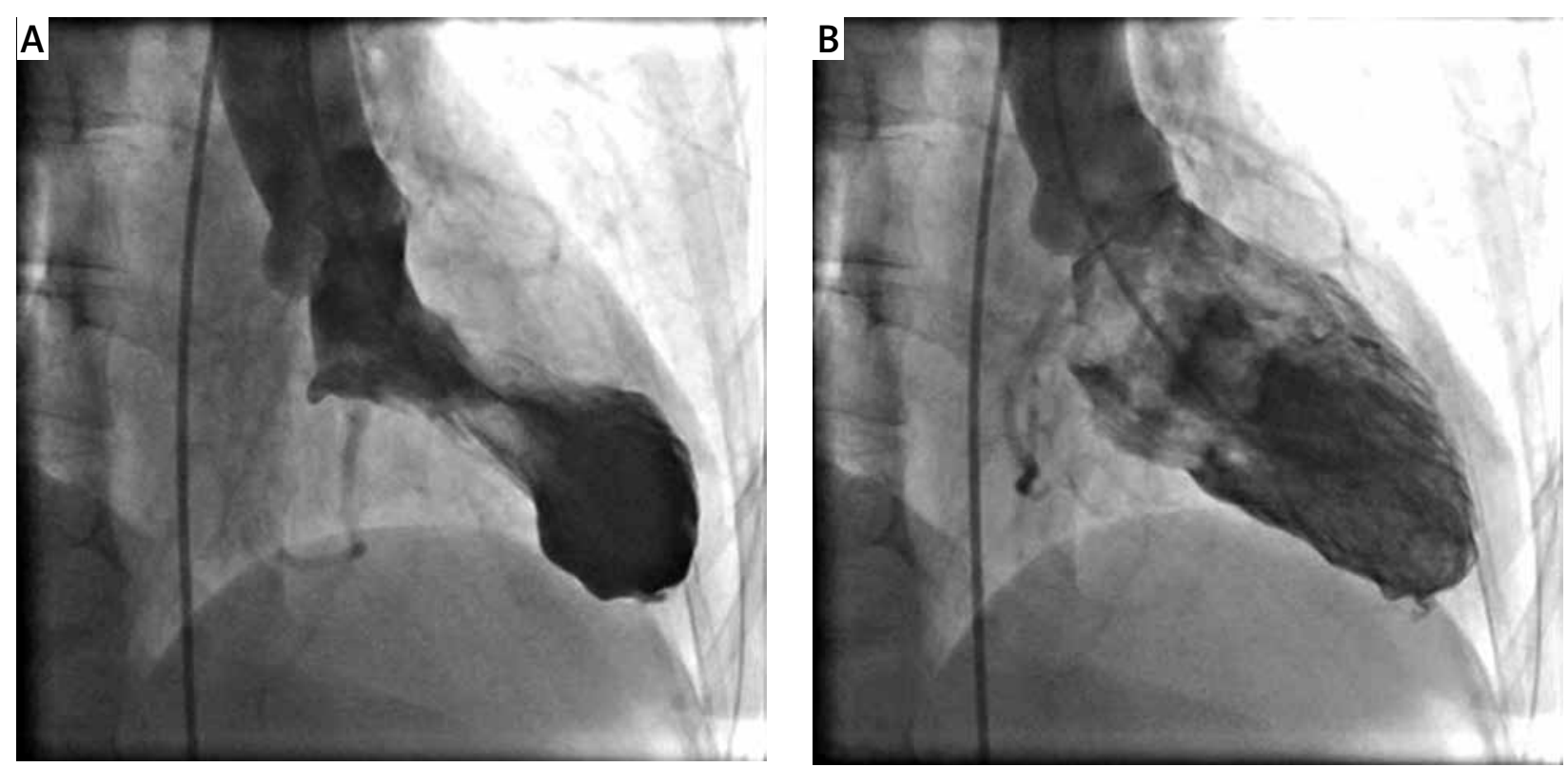

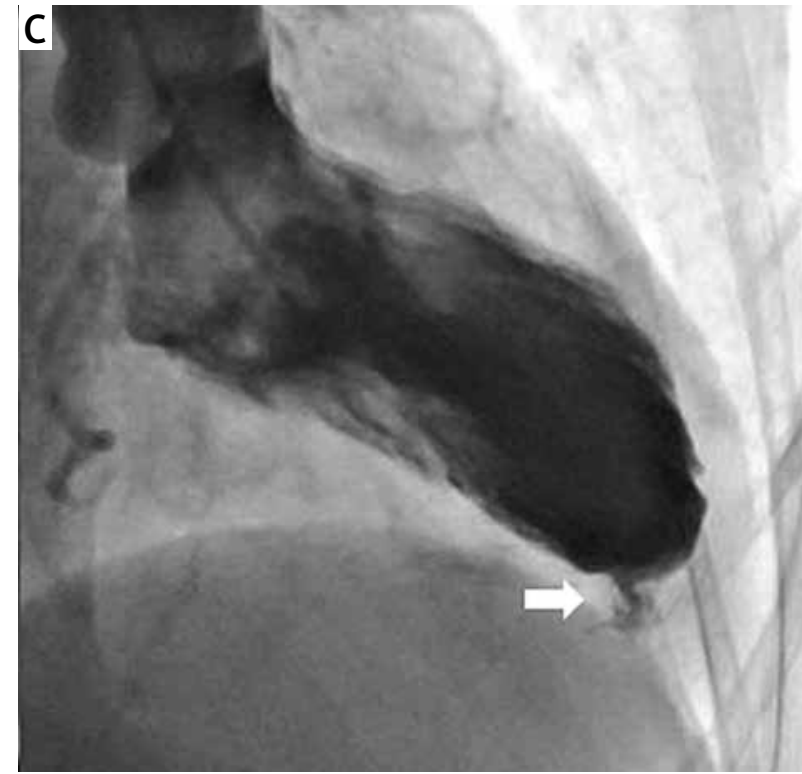

The present case provides evidence of the significance of monitoring patients during the first days of the TTC syndrome. Only immediate diagnosis and urgent cardiosurgical intervention leads to a favorable prognosis in patients with TTC cardiomyopathy complicated by cardiac rupture.

\section{Conflict of interest}

The authors declare no conflict of interest.

\section{References}

1. Kumar S, Kaushik S, Nautiyal A, et al. Cardiac rupture in Takotsubo cardiomyopathy: a systematic review. Clin Cardiol 2011; 34: 672-76.
Figure 1. A - ventriculography (left ventricular systole - normal volume of the left ventricle, contractile disturbances of the apex and hyperkinesis of the basement segments), B - ventriculography (left ventricular diastole), C - ventriculography (contrast outflow within the area of the apex white arrow)

2. Templin C, Ghadri JR, Diekmann J, et al. Clinical features and outcomes of Takotsubo (stress) cardiomyopathy. N Engl J Med 2015; 373: 929-38.

3. Sosnowska-Pasiarska B, Bąkowski D, Woronowicz-Chróściel A, et al. Sudden cardiac arrest in takotsubo cardiomyopathy a case study. Postep Kardiol Inter 2014; 10: 110-3.

4. Zawiślak B, Dziewierz A, Kmita A, et al. Ventricular septal rupture in a patient with non-ST-segment elevation myocardial infarction caused by myocardial bridge. Pol Arch Med Wewn 2015; 125: $386-8$ 